Alma Mater Studiorum - Università di Bologna DEPARTMENT OF ECONOMICS

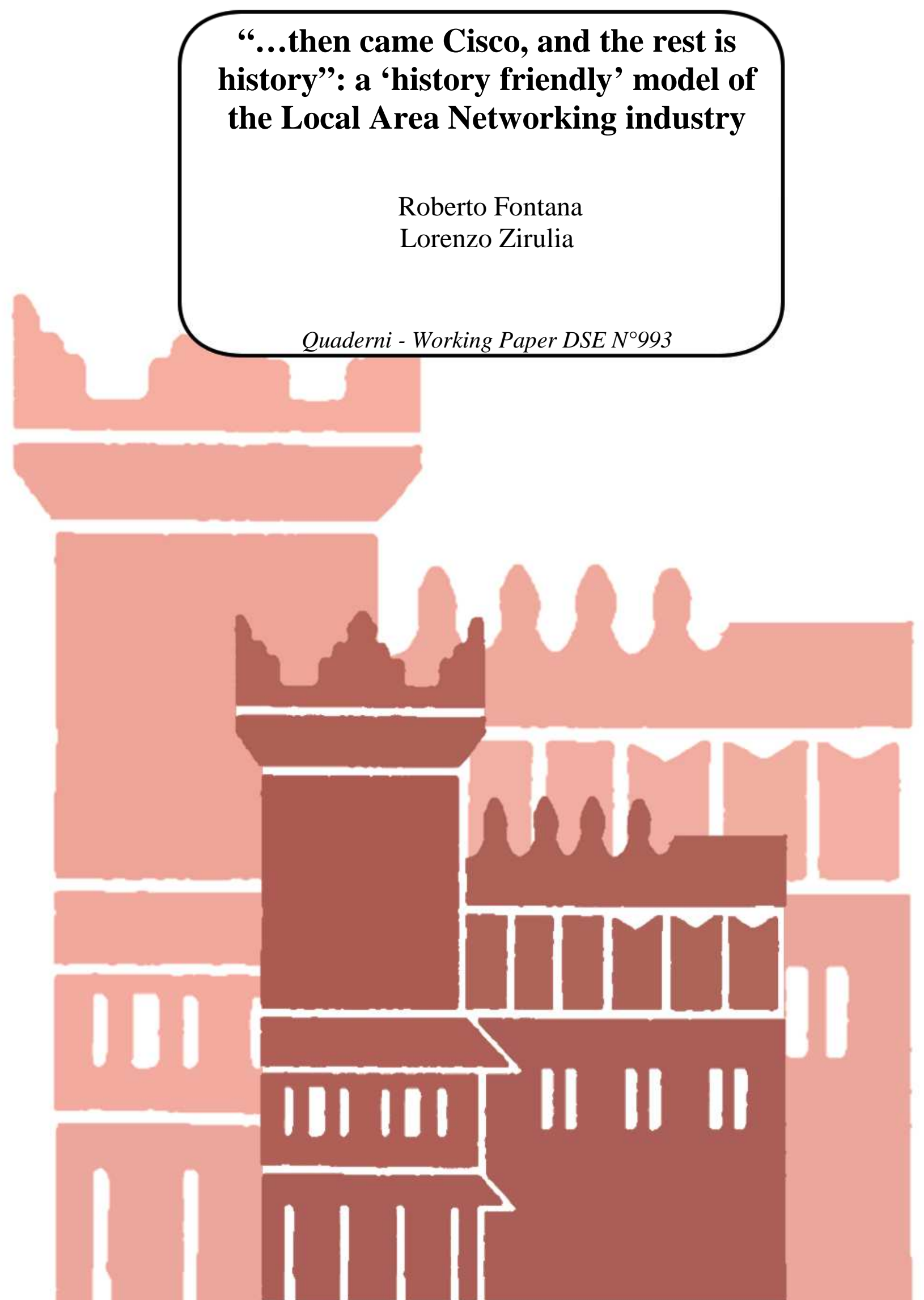




\title{
"...then came Cisco, and the rest is history": a 'history friendly' model of the Local Area Networking industry
}

\author{
Roberto Fontana*, Lorenzo Zirulia**
}

February 9, 2015

\begin{abstract}
We study the role that switching costs, compatibility, and mergers and acquisitions, play in influencing the evolution of a multi-market industry. By looking at the case of the Local Area Networking industry, we propose a 'history friendly model' to replicate its evolution during the 1990s. Our model explains how a firm can start from a dominant position in one of the existing markets and exploit switching costs and compatibility to enter a new market when it opens. Mergers and acquisitions also play an important role as the new market is pioneered by a new start-up, which is soon acquired by the dominant incumbent. As a result of the acquisition, the acquiring firm becomes leader also in the new market.
\end{abstract}

Key words: Simulation, History Friendly Models, Switching Costs, Compatibility JEL classification: 030, L10, L63

\footnotetext{
* Department of Economics \& Management, University of Pavia, Via San Felice 5, 27100, Pavia, Italy and CRIOS Bocconi University, Via Sarfatti 25, 20139, Milan, Italy. Tel: +39 02583630 37; Fax: +39 0258363399 Email: roberto.fontana@unibocconi.it

** Department of Economics, University of Bologna, CRIOS - Bocconi University and RCEA. Email: lorenzo.zirulia@unibo.it
} 


\section{Introduction}

The aim of this paper is to propose a 'history friendly' model of the Local Area Networking (LAN) industry in the 1990s. LANs are physical infrastructures enabling computers, peripherals and more in general 'end stations', to be linked to form a network connecting different users within an area of narrow extension (i.e. a university campus or different buildings at a company site). The LAN industry is part of the data communication industry that accounts for $30 \%$ of the total spending on communications equipment in the US. During the period considered in this paper, which corresponds to the phase of maximum growth of the industry, spending on LAN equipment accounted for $47 \%$ of the total for data communications and $14 \%$ of the total for telecommunication equipment in US (Doms, 2003).

'History friendly' models (HFMs from now on) are conceived as part of a second generation of evolutionary models of industry evolution. While first generation models (Nelson and Winter, 1982) were abstract and general, primarily aiming at exploring the logic of evolutionary economic processes and at establishing the utility of this new approach, HFMs intend to focus on and to explain the historical evolution of specific industries. The first example of a HFM was proposed by Malerba et al. (1999), who also provided a discussion of the HFM methodology. ${ }^{1}$

Our paper focuses on the structural dynamics and evolution of the LAN industry during the 1990s, an extremely dynamic period in which growth occurred as the consequence of the interplay between technical change and new market creation. The evolution of the LAN industry proceeded in phases, each of them characterized by the presence of several markets (one for each piece of LAN equipment) and each phase characterized by the presence of a 'focal' market. During the 1990 's, Cisco Systems was able to become the dominant firm in the LAN industry by extending its dominance from an existing market (routers) into a new market (switches).

The appreciative theory we propose to explain this pattern is based on three elements: switching costs, compatibility, and mergers and acquisitions (M\&As). In the LAN industry, switching costs derived mainly from the need to purchase equipment that was compatible with the existing installed base, and from the extra cost customers incurred to learn how to use equipment purchased from different manufacturers. LAN equipment 'work together' on the basis of common and open standards (Jain, 2012). However, all the major industry leaders developed families of

\footnotetext{
${ }^{1}$ Malerba et al. (1999) presents a model of the evolution of the computer industry. Other sectors which have been studied using this approach include pharmaceuticals and biotechnology (Malerba and Orsenigo, 2002), and the DRAM industry (Kim and Lee, 2003).
} 
equipment and dedicated software platforms in an attempt to differentiate their offer from those of competitors (Gawer and Cusumano, 2002). In this context, compatibility (or lack thereof) became an important issue to maintain market leadership. M\&As of new start-ups where also used by incumbents to restrain competition and gain access to new technological capabilities. In a nutshell, we argue that Cisco Systems was able to become the industry leader by exploiting its installed base, through switching costs and compatibility, while simultaneously acquiring new technological capabilities through a mix of targeted M\&As (Mayer and Kenney, 2004).

Our formal model captures all the distinguishing features of this appreciative theory, and it produces results that are in line with the observed history. In particular, the observed high level of concentration and a high market share for the leader in all the markets is obtained only for positive level of switching costs. M\&As also play a crucial role in shaping the industry evolution, as a counterfactual exercise excluding them produces an evolution of the industry that is rather different from the observed one.

Alongside the contribution of our modelling exercise, our work contributes to a recent stream of literature which studies the co-evolutionary dynamics of related markets (Malerba et al., 2008; Malerba and Orsenigo, 2010; Bhaskarabhatla and Klepper, 2014). We extend this literature by considering the case of co-evolution of markets for complementary goods in presence of 'frictions' such as switching costs and partial compatibility. Other industries (for instance software/hardware in IT) present similar characteristics and some of the dynamics we describe are likely to be observed also in these industries.

The paper is structured as follows. Next section provides the background information on the evolution of the LAN industry. In particular, it identifies those facts of industry evolution which we intend to focus on, and sets the stage for the development of an appreciative theory in Section 3. Section 4 briefly presents the HFM methodology, discusses its aims and highlights its limitations. The HF model is then described in Section 5. Section 6 reports the results from numerical simulations and presents first history-replicating runs, followed by few historydivergent experiments. Section 7 discusses the findings in terms of their contribution to recent literature on industry evolution. Section 8 concludes. 


\section{The evolution of the LAN industry}

\subsection{Overview and definitions}

This section presents an overview of the main events that have characterized the pattern of innovation and the evolution of the LAN industry with specific reference to the 1990s the years or rapid growth and consolidation. It provides the necessary background information for understanding the model and the analysis that will be carried out in the rest of the paper.

LANs constitute the physical infrastructure for data communication across an area of narrow extension (e.g. a university or company). Over LANs data travel in packets from a possible sender to a data receiver according to some 'rules' defined by standards. Alongside standards, the infrastructure of modern LANs is made up of several types of equipment which govern the data traffic at different levels of technological complexity and hierarchy. We focus on three types of equipment: hubs, routers, and switches. Within a LAN, each piece of equipment plays a specific role. Hubs are very simple equipment which are used to extend networks. Routers are technologically complex as they embody the algorithms used to optimally transfer data across users and between separate LANs. Switches are used to accelerate data transfer and processing. They are located between hubs and routers in terms of technological complexity. The wiring of hubs, routers, and switches form a 'system'. LAN firms manufacture and commercialise the pieces of equipment that constitute the system. Each type of equipment is commercialised in a specific market of the industry and LAN manufacturers may or may not be active in every market. During the evolution of the industry, and as a consequence of technological change, the functionality of equipment changed and the boundaries between the markets have progressively blurred.

The diffusion of office LANs started in the second half of the 1970s. Early LANs were essentially 'company' networks based on proprietary technologies (i.e. the Xerox Network and the DEC Network etc.). An acceleration in the diffusion of LANs within office environments occurred during the first half of the 1980s as a consequence of the official definition of Ethernet and Token Ring as LAN transmission standards. Both Ethernet and Token Ring enabled companies to connect the sparse existing 'legacy' LANs by using two types of equipment: bridges and routers. Bridges and routers were mainly used to connect networks running incompatible standards. Hubs were introduced to rationalize cable deployment and enlarge existing networks. At the end of the 1980s, the process of enlargement created congestion problems as new and more performing computers were adopted in company offices and new 'bandwidth hungry' voice and video applications became available. Two possible solutions slowly emerged. The first was the definition of new high speed standards which could enable data packets to travel at higher speed than that provided by 
Ethernet and Token Ring (10Mbps and 4 Mbps respectively). The second was the deployment of switch equipment that progressively substituted for hubs within high speed LANs (Christensen et al., 1995).

The definition and commercialization of high speed standards that occurred in the first half of the 1990s saw initially the competition between different alternatives (Fast Ethernet, FDDI, 100VGAnyLAN and ATM) and ended in mid-1990s when Fast Ethernet became dominant (Fontana, 2008). The diffusion of new standards and the opening up and evolution of new markets were not disjointed. The definition of new standards at high speed represented for LAN users a possible solution to congestion problem but, at the same time, a new source of possible bottlenecks when the implementation was not accompanied by changes in the design of the equipment. During the 1990s, technical change in hub equipment mainly consisted in changes in the hardware design in response to the introduction of high speed standards. This dynamic sustained the growth of the market. A further evolution consisted in the development and introduction of switch equipment. Switches embodied many of the functionalities played by hubs and threatened their existence. This happened during the second half of the 1990s which witnessed the progressive decline of importance of the role of hubs within LANs and the fast diffusion of switches while the router market stood still. The changes in revenues reported in Figure 1 below reflect the events just summarised.

\section{[Insert Figure 1 about here]}

We now analyze more in detail the dynamics of the two key markets in the 1990s, routers and switches. $^{2}$

\subsection{The router market}

Starting in 1989 the router market has passed through three phases, each opened up by some kind of technological innovation (either radical or incremental). The introduction of the 'multi-protocol function' was the event that opened up the market and engaged router manufacturers in close competition. The market then entered a diversification phase (1992-1994) in which incumbents started to offer 'scaled down' versions of previous products (i.e. remote-access routers) following

\footnotetext{
2 Existing contributions have provided detailed evidence on the early history of the industry (von Burg, 2001) and on the events that have characterized the most recent period especially concerning the optical networking market of the data communication industry (Carpenter et al., 2003).
} 
mainly two different innovative strategies, software-only routing or hardware-based routing. ${ }^{3} \mathrm{~A}$ new phase started in 1995 when router manufacturers felt progressively threatened by new entrants coming from the switch market (Miller, 1996).

During all these phases the structure of the router market did not change much. Entry and exit combined with decreasing prices occurred. Market shares and firms' ranking, considering also M\&As, remained relatively stable though a look at the changes in market shares for the overall market between 1990 and 1996 (see Table 1) reveals a tendency toward a growing concentration.

\section{[Insert Table 1 about here]}

Contrasting this relative stability there was much more turbulence at the sub-market level (i.e. multi-protocol and remote-access router). During the period of diversification total revenues in both sub-markets continued to grow and average prices to fall, but at different rates in each submarket. Prices of multi-protocol routers decreased relatively less than in the remote-access submarket.

Competition was less effective in the case of the multi-protocol sub-market because of higher barriers to entry represented by both the high capabilities required to provide the software responsible for the multi-protocol routing function and the presence of demand side switching costs. Most of new firms which entered the sub-market during the diversification stage could not provide the routing function in their products which had to be handled by PCs. Multi-protocol incumbents responded to the challenge of new entrants with new products that contained proprietary software and hardware thus rising demand side switching costs and barriers to entry (Miller, 1994). Prices for remote-access routers fell both because of competition from multi-protocol incumbents, and because of new entrants from the switch market.

In 1993, when the switch market took off, some switch manufacturers challenged existent incumbents also at the low-end of the router market. Thus, new entries in the remote-access submarket continued to increase fuelled by new manufacturers. As a result, within the router market the multi-protocol sub-market was systematically more concentrated than the remote-access submarket (see Table 2).

\footnotetext{
${ }^{3}$ Multi-protocol routers embody several algorithms and support several communication protocols to intelligently deliver data packets to their destination. Access-routers provide customers with an interface between their end stations and the network.
} 
[Insert Table 2 about here]

Competition in multi-protocol sub-market increased as the result of new entrants which, however, failed to challenge the leaders. A mix of technological advantage and the presence of demand side switching costs partially sheltered incumbents from increasing competition. The remote-access sub-market instead suffered the consequences of two types of competition. Increased competition from outside the market was as effective as intra-market competition in disrupting technological barriers.

\subsection{The switch market}

The first LAN switch was developed in 1990 by a US start-up company: Kalpana. From the technological viewpoint, early switches were an incremental innovation with respect to early bridges that had been previously developed to interconnect LANs using different proprietary standards. Thus when they first appeared in the market they engaged in a direct competition with bridges rather than with routers. Second generation switches incorporated technological improvements which enabled them to perform more sophisticated data communications thus challenging what until then had represented exclusive application domains of routers.

From an economic viewpoint, the introduction of the LAN switch had a great impact on the structure of the industry. A sign that the new product had fulfilled a latent demand for more bandwidth in the market was an immediate increase in sales aided by falling prices. As a result, in 1997 a typical switch was priced (on average) only 35\% more than a remote-access router. The price premium was even smaller as switches could ensure higher data speed and performance. The commitment of suppliers to address demand came together with a change in the structure of the market, although changes were different from the ones experienced by routers (see Table 3 below).

\section{[Insert Table 3 about here]}

The arrival of the switch impacted deeply on the sales of the other LAN products. The LAN switch was a definite hit for LAN bridges and routers sales slowed down. Also, switches represented a window of opportunity for a swarm of new firms to enter the industry, attempt to undermine, for the first time since its foundation, the growth of the router market, and to lay foundations for future changes in the entire industry. 
The structure of the switch market has always been concentrated, although notably less than that of routers. In 1994, the four biggest firms in the market (Cisco Systems (Kalpana), 3Com (Synernetics), Alantec and Chipcom) accounted for 94\% of revenue share. In the 2nd quarter of 1999, the share of the four biggest companies had fallen to $81 \%$, with Cisco Systems maintaining the lead at $47 \%$, followed by 3Com, Nortel Networks (Bay Networks) and Cabletron. Contrary to what had happened in the router market where there were no major changes in firms' ranking, here the market experienced a marked shuffle. This difference is the result of the different dynamics underpinning entry and exit in this market when compared to routers'.

Entry in the switch market was driven by three types of manufacturers. First, there were incumbents from within the LAN industry (both from the router and the hub market). Cisco Systems, the future dominant firm in the switch market, was originally a router maker and was among the first firms to enter. More hesitant incumbents in the hub market (3Com, Bay Networks, DEC) were constrained by their previous investments as well as by the risk of cannibalizing their installed base. Second, there were incumbents from outside the industry but with previous experience either in the telecom industry or in the semiconductor industry. Third, there were startups searching for new opportunities.

Consolidation occurred mainly because of two reasons. First, incumbents strengthened their position through a mix of targeted M\&As. Cisco Systems, soon to extend its dominance from the router into the switch market, entered by acquiring Kalpana the market leader and pioneer of the technology. In 1994, SynOptics (a hub maker) merged with Wellfleet (a leader in the router market) to create Bay Networks. Indeed entry by acquisition soon became one of the main features of the LAN industry and several recent works have investigated both the economic (Fontana and Nesta, 2009) and the managerial/organizational (Paulsen, 2001; Mayer and Kenney, 2004) implications of this strategy of growth.

Second, incumbents leveraged on their existing leadership in related LAN markets to become dominant in the new switch market. This strategy was facilitated by the peculiar nature of the switch market which soon became polarized. Indeed, the growth of the switch market was accompanied by developments in the equipment design (i.e. transition to Application Specific Integrated Circuits based hardware). As a result of these events, in the second half of the 1990s, two sub-markets opened up. High-end switches, characterized by high performance, were targeted to customers with large networks. Low-end switches were low cost and generally less 
performing, supported only one standard and were targeted to customers with small networks (Fontana and Nesta, 2006).

\section{Towards an appreciative theory}

The previous sections have synthesized the main events and facts that have characterized the structural dynamics and evolution of the LAN industry during the 1990s. The aim of this section is to highlight these facts and to develop an appreciative theory to be formally represented in a HFM.

The first fact emerging from the previous section is that the evolution of the LAN industry proceeded in phases each of them characterized by the presence of several markets (one for each LAN product) and each phase characterized by the presence of a 'focal' market. The opening up and subsequent growth of the 'focal' market was usually the consequence of the interplay between innovation and changes in customers' needs which led to the progressive redefinition of the LAN structure through time. In each phase major firms competed to become dominant in the 'focal' market.

The second fact is that dominance was usually achieved through two types of competition. First, there was inter-market competition which led LAN incumbents to enter into related markets of the industry. This is the competition that occurred along the 'vertical structure' of the industry and was facilitated by technological change that made products more and more similar in terms of functions. We define this type of competition: 'technological competition'. Second, there was the competition that occurred within a specific market (i.e. intra-market competition). The aim of this type of competition was to differentiate equipment within each market in order to make the demand increasingly inelastic. We define this type of competition: 'demand competition'. While technological competition blurred the functional boundaries across products, demand competition led to fragmentation with technological variety that increased both horizontally (i.e. inter-firm market distance grew) and vertically (i.e. the variance of distance from frontier grew as markets became polarized).

The third fact is the role played by new entrants vis-à-vis incumbents in shaping the industry structure. The opening up of a potential 'focal' market triggered a swarm of new entrants, initially start-ups or spin-outs, who could supply new equipment, improve upon an existing one, or compete with incumbents by mean of differentiation first and then price competition. Confronted with the entry of new firms, incumbents reacted in different ways. In particular, a mix of targeted $M \mathcal{E} A s$ was one of the strategies pursued by incumbents to obtain the needed technological 
capabilities. In the period our model aims to represent, the 1990s, the switch market emerged as the new focal market. Kalpana, a start-up, pioneered the technology and become the market leader; soon, however, it was acquired by Cisco Systems, which was able to extend its dominance from the router into the switch market.

Cisco Systems was by no means the only LAN manufacturer who grew through M\&As. What we suggest is that Cisco's M\&As' strategy turned out to be successful for the concurrent presence of switching costs and compatibility strategies. In the LAN industry, switching costs derived mainly from the customers' need to purchase equipment that was compatible with their existing installed base, and from the extra cost of learning to use equipment purchased from different manufacturers. Manufacturers exploited the presence of high switching costs to lock-in customers and to compete against rivals in several ways, for instance by strategically setting the timing of new product introduction to force customers to buy upgrades. In their analysis of the determinants of switch equipment choice by users, Chen and Forman (2006) present evidence that the presence of an installed base of equipment from a particular manufacturer increases the likelihood of repurchasing from the same vendor if the customer decides to buy again. These issues become particularly important in the case of technologically sophisticated equipment such as high-end switches used in large networks of hundreds or thousands of users.

As far as compatibility is concerned, LANs developed in an environment characterised by open and common standards (Jain, 2012; von Burg, 2001). In this context, customers in theory can mixand-match equipment from different manufacturers in the same network. In practice, firms adopted several strategies to 'close' their system. First, switches need software program to function and manufacturers often designed proprietary software that made their equipment incompatible with that of other manufacturers. For this purpose, Cisco Systems developed the 'Cisco Fusion' architecture first and then the 'Internet Operating Systems' (IOS) software (Gawer and Cusumano, 2002). ${ }^{4}$ Second, manufacturers introduced families of products that worked well together. These families typically spanned across different categories of users so as to be able to target the entire demand spectrum (i.e. going 'end-to-end') and/or to prevent rivals from entering. In 1994, Cisco Systems offered the first product of its Catalyst line of Switch equipment that was enriched in the following years by other high-end as well as low-end equipment. ${ }^{5}$

\footnotetext{
4 In a similar move, Cabletron Systems introduced the 'Spectrum' network management software.

${ }^{5}$ Again major competitors did not stand still. 3Com responded with the Superstack and Office Connect line of equipment, the former targeting big users, and the latter customers with smaller networks. Bay Networks and Cabletron Systems, marketed an entire new product line: the BayStack and Smartswitch, respectively.
} 
Both switching costs and compatibility (or lack thereof) provided incumbents a comparative advantage in terms of installed base, to be exploited once technological capabilities were acquired through targeted acquisitions. As an alternative strategy to maintain leadership, incumbents could try vertical differentiation (i.e. pushing forward the technological frontier). Since the blurring of functional boundaries across equipment represented a limit to the growth of a specific market, the technological frontier in the specific 'focal' market has to be moved forward for the firm to continue to gain rents. However, data suggest that incumbents were able to maintain their leadership without necessarily competing at the technological frontier. For each market in selected years, Table 4 below ranks manufacturers in terms of their location with respect to the technological frontier.

\section{[Insert Table 4 about here]}

Seen from a different perspective, Table 4 suggests also that being at the technological frontier does not seem to be sufficient for becoming dominant in the industry. Cisco Systems in both the router and the switch market is the most striking example. This evidence suggests also that new entrants lacked other distinguishing features to challenge the leader: the possibility to leverage upon demand side switching costs and 'system closure' strategies.

The formal model we will develop in the next section captures all the essential elements of the appreciative theory outlined above. Innovation takes the form of new product introduction, both within existing markets and in new ones. New products are introduced both by incumbent and new firms. On the demand side, customers incur switching costs when changing suppliers, and products produced by different suppliers may be partially incompatible. Finally, M\&As between firms that are complementary in terms of their product portfolio are another distinctive feature of the model.

\section{The HF methodology: aims and limitations}

Malerba and Orsenigo (1996) identify three dimensions of industrial dynamics: i) 'industrial demography' (i.e. specific dimensions of industrial dynamics such as entry rates and size distributions); ii) 'structural dynamics' (i.e. the joint dynamics of the main structural variables characterizing an industry such as entry and exit rates, market concentration, innovation and profitability); iii) 'structural evolution' which considers the evolution of an industry in a broader perspective, which includes factors as different as the emergence of new products and 
technologies, learning modes and capabilities, changing boundaries of firms and the role of policies, institutions and public organizations.

From a substantive point of view, HFMs are motivated by the recognition that any explanation of structural evolution of industries is almost by definition industry-specific, relying on the details of the sectoral system of innovation and production (Malerba, 2002). The most common way to analyze the structural evolution is through appreciative theorizing, which is defined as the nonformal explanation of observed phenomena based on specific causal links proposed by the researcher (Nelson and Winter, 1982). Although appreciative theorizing must be seen as 'true' causal theory and a fundamental process of building a theory, it can be difficult to assess if the suggested causal arguments are consistent and sufficient to provide an explanation, in particular if the phenomena under investigation embody non-linear and path-dependent processes.

Malerba et al. (1999) argue that the primary goal of HFMs should be to verify the logical consistency of appreciative theorizing put forth by analysts of the history of an industry or a technology. Being rooted in the evolutionary tradition, HFMs do that by relying on the typical ingredients of agent-based models, in which aggregate outcomes are understood as the emergent property of interaction among heterogeneous and boundedly rational agents. Therefore, a first objective of the HFM exercise is to assess if the proposed chain of causal links together with a set of 'reasonable' values for the parameters, is able to replicate the actual evolution of the industry, achieving what Malerba et al. (1999) have defined as history-replicating simulations. If that is the case, the capacity of the formal model, and indirectly of the appreciative theorizing, to be a valid explanation of the empirical evidence is supported. In our set-up, this approach will consist in showing that a model along the lines described in previous section, in which i) switching costs exists; ii) compatibility is not full and iii) mergers and acquisitions are used by firms to acquire capabilities and enter new markets, is actually able to reproduce the facts we identified. This exercise is accomplished in Section 6.1.

Once after achieving this aim, the model can then be used to analyze to perform counterfactual analysis, in order to look for what Malerba et al. (1999) called the 'history-divergent runs'. Counterfactual analysis is frequently conducted, although often implicitly, by social scientists and historians, but at the same time, the conditions for its validity has been significantly debated (Tucker, 1999). In its general formulation counterfactual analysis consists in the falsification of the antecedent in order to investigate the consequent. In their discussion on the use of counterfactuals as empirical tool for evolutionary economics, Cowan and Foray (2002) identify two particular 
views of the counterfactual put forth in the philosophical literature. The first one, associated to Lewis (1973), sees counterfactuals as propositions related to 'worlds' that, although possible, are different from the one that actually exists. The second view, associated to Elster (1978), understands history as a tree in which each decision represents a branching points, leading to new decisions and new branching points as time elapses. In this case, performing counterfactual analysis means 'moving backwards' in the decision tree and taking a different decision, leading to a different branch, at some point. This view is somewhat more appealing, or at least intriguing, for scholars in the evolutionary tradition, for which path-dependence and the role of history in the selection of trajectories (if not equilibria) is important. At the same time though, this approach poses more subtle issues on the validity of the exercise in terms of the ancillary assumptions one needs to make. 6

In the HFM tradition, we find examples of counterfactuals that in fact adhere to both view. Within the 'possible worlds' view of counterfactuals we find exercises of 'comparative dynamics' in which key parameters are varied. In Malerba et al. (1999), bandwagon is seen as fundamental part of the explanation for the different evolution of the mainframe market with respect to the PC market (being bandwagon effects stronger in mainframes than in PC), so that ruling out bandwagon effects, in particular for mainframes and for all simulation horizon in fact leads to history divergent runs. ${ }^{7}$ Within the branching view of counterfactuals, instead, one can include those experiments trying to verify the effect of specific public policies (which can be located in time) on industrial evolution. For instance, Malerba et al. (2001) analyze the importance of timing in the antitrust policy implementation towards the near monopolist in mainframe (IBM).

In our paper we exclusively consider counterfactuals of the former type, by assuming out switching costs, partial compatibility, and M\&As. Obtaining history-divergent outcomes, within the same system of causal links, we find further support for out theory and we obtaining additional theoretical insights. Moreover, we also experiment with other mechanisms for industrial evolution, in particular related to increasing returns on the supply side, finding that they alone can explain some facts of industry evolution, but not all of them.

Though quite consolidated in the literature, it has to be noted that HFMs have been criticised within the debate on the empirical validation of agent-based models in economics (Fagiolo et al., 2007). The central issue, we believe, concerns the extent to which an in-depth description and

\footnotetext{
6 See Cowan and Foray (2002) and Tucker (1999) for a detailed discussion of this point.

7 See the review of Garavaglia (2010), Section 4.2, for a selection of other similar counterfactual exercises.
} 
simulation of an individual case can be helpful in drawing a generalizable set of conclusions about industrial dynamics. Put it differently, it has been argued than being able to build a model which replicate the actual history as one of the possible outcomes is not in fact a very strong test. Nor, we should accept or reject a theory based on how typical the single, observed history is within the model generated data.

With respect to the latter criticism, we do agree that the problem of identification (i.e. several alternative explanation for the same phenomenon) is 'by construction' particularly serious for HFMs, although it appears frequently in other types of agent-based models, where the number of parameter assumptions, and consequently the degrees of freedom, tend to be high. However, if one accept the view that structural evolution is both industry-specific and worth of explanation, as we do, then HFMs are in fact the only available alternative to check the validity of the appreciative theory by explicitly spelling out the involved mechanisms and assumptions and therefore verify the soundness of the economic argument.

In addition to this, we think that HFMs and other more general evolutionary models of industry evolution such as Winter et al. (2003) and Bottazzi et al. (2001) among many others, should be seen as complementary rather than substitute. In fact, the latter typically focuses on the second dimension of industrial dynamics, i.e. structural dynamics, for which one can indeed assume that what is observed in different sectors are realizations of the same 'real-world data generating process' (in the terminology of Fagiolo et al., 2007).

Also, we think that HFMs may fruitfully lead to models of more general applicability, i.e. relevant for other industries as well. This can be done in two ways. First, based on the original HF exercise, extensions of the model may be used to investigate issues of more general interest. This is what is actually done in Garavaglia et al. (2012), where an improved version of the HFM of the pharmaceutical industry developed in Malerba and Orsenigo (2002) was used to investigate how technological regimes and demand structure can interact in determining market structure. Second, the result of a specific HFM may contribute to a literature, or to the debate, on more general theme in industrial dynamics, for instance by qualifying existing results or identifying cases previously neglected. This is exactly what our contribution does. In Section 7 we will see how our results can be interpreted in the light of, and contribute to, the literature on the co-evolutionary dynamic of related industries. In addition to this, they can also provide insights for other modelling approaches notably in mainstream industrial organization. 


\section{The model}

The set-up of the model can be described as follows. There are two categories of agents: customers and producers. Customers are firms that buy products (i.e. LAN equipment) to build their system. Producers manufacture and sell the products that constitute a system. Simple rules drive the changes in the product line of manufacturers over time.

\subsection{Products}

Over the simulation horizon, we consider three types of products, type 1, type 2, and type 3. Each product is described in terms of its quality level $(q>0)$. Products are used to build systems. Each system is described by an architecture, which consists in a set of products and weights associated to each product. We consider two possible architectures, $A$ and $B$. The quality of the system, which is determined by the quality of its constituent products and weights, enters into the utility function of customers. Formally, we use $y$ to denote a generic system, and $Y$ to indicate the set of all the systems available at a given moment in time. We denote with $q(y)$ the quality of the system, and $q_{k}(y)$ the quality of the product of type $k$ in system $y$.

Architecture $A$ consists of one product of type 1 and one product of type 2 . The overall quality of the system can be expressed by a Cobb-Douglas function with weights $a$ and $(1-a) .8$

$$
q(y)=\left(q_{1}(y)\right)^{\alpha}\left(q_{2}(y)\right)^{1-\alpha}
$$

Architecture $B$ is constituted by three products, one by each type. In this case, the weights of the Cobb-Douglas function are $\beta_{1}, \beta_{2}$ and $1-\beta_{1-} \beta_{2}$ :

$$
q(y)=\left(q_{1}(y)\right)^{\beta_{1}}\left(q_{2}(y)\right)^{\beta_{2}}\left(q_{3}(y)\right)^{1-\beta_{1}-\beta_{2}}
$$

At time 0 , only architecture $A$ is feasible (i.e. manufacturers cannot produce type 3 products). At time $\bar{t}$ manufacturers can produce type 3 products and therefore architecture $B$ becomes available. Our assumption is that architecture $B$ is better than architecture $A$, in that it allows reaching quality levels that are impossible to reach under architecture $A$. Architecture $A$ resembles early LANs in which buyers could combine only two types of equipment (i.e. routers and hubs).

\footnotetext{
8 This formulation is consistent with Malerba et al. (1999) who assume that the quality of the final product is a C.E.S. function of the quality of different products.
} 
Architecture $B$ becomes viable when also switches are commercialised. The mechanisms determining products quality are described in Section 5.2 .2 below.

\subsection{Manufacturers}

The number of potentially active manufacturers is exogenously given and fixed the beginning of the simulation horizon. At the beginning of each period, each manufacturer has a product portfolio made of several products (this portfolio is assumed to be empty at $t=0$ ). In each period, product portfolios are modified following two simple rules concerning:

1. Mergers and acquisitions;

2. Innovation (i.e. the introduction of new products).

\subsubsection{Mergers and acquisitions}

At the beginning of each period, M\&As may occur according to a two steps procedure. In the first step, pairs of potentially merging manufacturers are formed. In the second step M\&As can occur with certain probabilities. To understand how M\&As occur, we proceed backward and assume that the pairs of potentially merging manufacturers have been formed. Denote with $s_{k i}$ and $s_{k j}$ manufacturer's $i$ and $j$ market share (in physical units) in the market for type $k$ product. The probability that the two manufacturers merge is given by:

If $t<\bar{t}$

$\operatorname{Pr}($ merger $)=0 \quad$ if $\left(s_{k i}-s_{k j}\right)>0 \forall k$ or $\left(s_{k j}-s_{k i}\right)>0 \forall k$

$\operatorname{Pr}($ merger $)=\theta\left|\max \left(s_{0 i}-s_{0 j}, \Delta s_{\min }\right)\right|^{\alpha}\left|\max \left(s_{0 i}-s_{0 j}, \Delta s_{\min }\right)\right|^{1-\alpha} \quad$ otherwise.

If $t \geq \bar{t}$

$\operatorname{Pr}($ merger $)=0 \quad$ if $\left(s_{k i}-s_{k j}\right)>0 \forall k$ or $\left(s_{k j}-s_{k i}\right)>0 \forall k$

$\operatorname{Pr}($ merger $)=\theta\left|\max \left(s_{0 i}-s_{0 j}, \Delta s_{\min }\right)\right|^{\beta_{1}}\left|\max \left(s_{1 i}-s_{1 j}, \Delta s_{\min }\right)\right|^{\beta_{2}}\left|\max \left(s_{2 i}-s_{2 j}, \Delta s_{\min }\right)\right|^{1-\beta_{1}-\beta_{2}}$

otherwise

where $\theta \geq 0$ and $\Delta s_{\min }>0$ and 'small'. The rationale underlying Equation (5.3) is that M\&As are more likely to occur between manufactures with high shares in different markets. M\&As are strategies of external growth, in which firms acquire new technological capabilities 'embodied' in products. This is in fact the role they played in the LAN industry. $\Delta s_{\min }$ sets a lower bound for 
positive probability of M\&As. In particular it allows M\&As to occur when the relevant architecture is $B$ and manufacturers are not active in all the markets.

To describe how M\&As occur in our model, we employ a rule similar to the one adopted by Cowan et. al. (2006) to describe the formation of bilateral strategic alliances in a model of knowledge creation. In the first stage, pairs are formed according to the following algorithm. The first pair of manufacturers is given by those manufacturers for which, according to Equation (5.3) the probability of merger is the highest. The second pair of manufactures is given by those manufacturers maximizing probability in Equation (5.3), excluding the manufacturers in the first pair. The third pair is formed by those manufacturers that maximize probability of merging according to Equation (5.3), excluding the manufacturers in the first two pairs and so on. ${ }^{9}$

\subsubsection{Innovation}

In each simulation period, after M\&As have possibly occurred, each manufacturer may change its product portfolio. With an exogenous probability $p$, the firm introduces a new product. The probability that the product is of a certain type $k$ is:

$$
\operatorname{Pr}(\text { type }=k)=\frac{\exp \left(\lambda_{1} n_{i k}\right)}{\sum_{k} \exp \left(\lambda_{1} n_{i k}\right)}
$$

where $\lambda_{1}$ is a positive parameter ${ }^{10}$ and $n_{i k}$ is the number of products of type $k$ offered by manufacturer $i$. According to Equation (5.4), firms are most likely to innovate in the markets in which they are already most active. This form of cumulativeness can be justified on the basis of idiosyncratic technological competences with specific products. The quality of the new product is drawn with uniform probability in the interval $\left(0\right.$, frontier $\left._{k}\right)$, where frontier $k_{k}$ is a time invariant

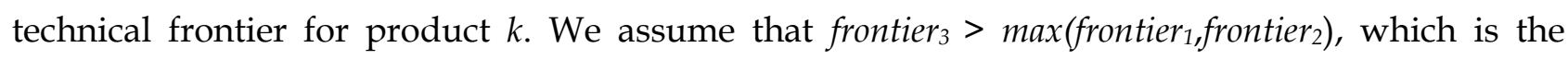
reason why architecture $B$ (which includes type 3 products) can achieve relatively higher quality levels.

The assumption that $p$ is exogenous may appear too extreme. Indeed, in most evolutionary models it is common practice to assume that the probability to innovate is endogenous and depends

\footnotetext{
${ }^{9}$ In those cases in which there is a 'tie' (i.e. we observe the same probability of mergers for all pairs in a subset of manufacturers), the first pair is formed by the manufactures with the highest indexes associated to each of them. The second pair is composed by the manufactures with the highest indexes associated to each of them, excluding the manufacturers in the first pair and so on. This 'tie-breaking' rule is adopted to save on computational time. Since ties basically occur when the probability of mergers is 0 , the tie-breaking rule is almost irrelevant.

10 The parameter $\lambda_{1}$ is known as the discrimination rate in the probabilistic choice rule.
} 
(positively) on previous innovativeness, profits or firm size. However, we believe that in our case it can justified on several grounds. First, from a purely theoretical view point, incentives for product innovation depend less than process innovation on firm size (Cohen and Klepper, 1996). Second, making this assumption allows us to focus on other specific mechanisms that have driven firms' growth in the LAN industry such as M\&As on the supply side, switching costs and imperfect compatibility on the demand side. Indeed, our account of the industry suggested that several firms (especially new start-ups) have played an important role as innovators, but their internal growth has been halted due to subsequent acquisitions by leading firms (Cisco Systems in particular), whose expansion was mainly a consequence of M\&As, and product innovation.

A manufacturer enters a market when it introduces its first product of a specific type. If the manufacturer's product portfolio is initially empty, entry into a specific market coincides with overall entry. If the firm is the first one to introduce a product of a certain type, firms' entry also coincides with the opening up of a new market.

\subsection{Customers}

The number of customer firms is exogenously given and fixed at the beginning of the simulation. In each period, with probability $\eta$, each customer is drawn from a distribution to purchase a new system. For simplicity, we assume that, once drawn, a customer simultaneously changes all the products constituting her system. $\bar{q}(y)$ denotes the 'actual quality' of the purchased system. This quality is the one described by Equations 5.1 and 5.2 only if: (i) the system is composed entirely by products supplied by the same firm or (ii) all products are supplied by firms that do not produce all the products which are feasible in a given moment in time. In all other cases, the 'actual quality' is given by $\bar{q}(y)=\gamma q(y)$, with $\gamma \leq 1 . \gamma$ captures the extent of compatibility across products. The idea is that manufacturers supplying customers with a full line of products can 'close' the system, as happened in the LAN industry through proprietary software.

$y_{i t}$ identifies the system bought by customer $i$ at time $t . y_{i t-1}$ indicates the system bought by the customer in the previous period. The utility customer $i$ benefits from buying $y$, when she had $y_{i t-1}$ in place is described by: ${ }^{11}$

\footnotetext{
11 This particular utility function borrows from Adner and Levinthal (2001), and many others, the idea that consumers differ in their marginal evaluation of quality. Differently from Adner and Levinthal, however, we do not explicitly consider prices. It can be noted, however, that Equation (5.5) can be considered as a reduced form of a case where price is determined by a fixed mark-up over cost, and unit cost of production is quadratic in quality.
} 
$U\left(y_{i t}, y_{i t-1}\right)=D_{i t} \bar{q}\left(y_{i t}\right)-\frac{1}{2} \bar{q}\left(y_{i t}\right)^{2}-\sigma_{i t t-1}$,

where $\sigma_{i t t-1}$ denotes a product switching cost which is equal to $\sigma \chi_{t t-1}$, where $\chi_{t t-1}$ is the number of products that are present in system $y_{i t}$ but not in system $y_{i t-1}$, and $\sigma$ is a positive parameter. $D_{i t}$ is a variable that represents the extent to which a customer is responsive to quality. In particular, it is easy to verify that it corresponds to the 'ideal quality' level for a specific customer, since it corresponds to the quality level that maximises $U$. $D_{i}$ increases over time according to the following expression:

$$
D_{i t}=D_{i 0}+\bar{D}(1-\exp (-g * t))
$$

where $D_{i 0}$ is uniformly distributed on $[0, \underline{D}]$ and $g$ is a positive parameter. The fact that $D_{i}$ grows over time is the reason why architecture B becomes attractive, first for consumers with high $D_{i 0}$ and then to the rest of customers.

The probability that $y_{i t}=y$ (i.e. the probability that a customer buys an available system $y$ ) is then given by: 12

$$
\operatorname{Pr}\left(y_{i t}=y\right)=\frac{\exp \left(\lambda_{2} U\left(y, y_{i t-1}\right)\right)}{\sum_{\tilde{y} \in Y} \exp \left(\lambda_{2} U\left(\tilde{y}, y_{i t-1}\right)\right)}
$$

\section{Results}

In this section we report the results from our numerical simulations. Following the HF methodology we first set out the conditions to obtain history-replication runs (Section 6.1), followed by few history-divergent simulations (Section 6.2).

In order to show that our model is able to replicate the observed dynamics, we impose positive switching cost $(\sigma>0)$, partial compatibility $(\gamma<1)$ and a positive probability for M\&As $(\theta>0)$. Instead of trying to calibrate $\sigma$ and $\gamma$ to an exact values, for each parameter we experiment with a

\footnotetext{
12 The exponential rule for representing consumer choices has been often used in models of industrial and market dynamics (Weisbuch et al., 2000).
} 
'low' and a 'high' value. For $\sigma$, the values are 1.5 (low switching costs) and 3 (high switching costs). For $\gamma$, the values are 0.6 (high compatibility) and 0.2 (low compatibility). ${ }^{13}$

In Section 6.2 we perform two types of counterfactual exercises. In the first counterfactual we modify the parameters model. In particular, we look at what happens when switching costs, partial compatibility and M\&As are excluded. Then, we analyze the case of homogenous demand. In the second counterfactual we consider a different mechanism for first-mover advantage (increasing returns), based on supply-side rather than on demand side dynamics.

Table 5 below summarizes the fifteen possible scenarios thus created. As for notation, we denote with $\operatorname{Scenario}_{(x, y, z)}$ the parameter setting in which $\sigma=x, \gamma=y, \theta=z$, and with Scenario $(H)$ and Scenario $_{(I)}$ respectively the case of homogenous demand and increasing returns based on supplybased considerations.

\section{[Insert Table 5 about here]}

Each run lasts 200 periods. Product 3 (i.e. Architecture B) becomes feasible at $\bar{t}=100$. For each scenario we considered 50 runs with different random seeds. We report the average value (across runs) of a set of indicators (specified below) measured at period 100 (when the new type of product is introduced) and at period 200 (when the run stops).

\subsection{History-replicating runs}

\subsubsection{Results for concentration and market leadership}

Table 6 reports the values of the Herfindahl Hirschmann Index (HHI) across markets over time.

\section{[Insert Table 6 about here]}

Results in columns (1) and (2) summarize the HHI for market 1 at the end of period 100 and at the end of period 200 respectively. It can be noted that, in each scenario, there is a tendency towards greater concentration over time, to reach a significant level, consistently with the observed history. The same dynamics is observed in the second market (columns (3) and (4)). This tendency is mainly driven by M\&As. In each market innovators and market leaders seek to exploit existing complementarities across products. One way to pursue this goal is through M\&As. The opening

\footnotetext{
13 The values of parameters that remain constant across simulations are reported in the Appendix.
} 
up of a new market creates new opportunities for exploiting complementarities and further triggers waves of acquisitions. As a result, firms increase their shares also in other markets.

This general dynamics notwithstanding, differences seem to exist across markets. In particular, for a given scenario, HHIs are usually higher for market 1 than for market 2 . In the case of market 3, HHIs are instead relatively low. In the case of markets 1 and 2 the result depends on the fact that the weight associated to product 2 in determining overall quality is higher (0.7 vs. 0.3). This implies that quality is relatively more important than other determinants of choices (i.e. switching costs and compatibility) for market 2 than for market 1 . As a consequence, market 2 is more competitive. As long as market 3 is concerned, the explanation for the difference lies in the different way in which purchases occur. While customers simultaneously purchase products from markets 1 and 2, purchase of type 3 products occurs sequentially provided that the marginal benefits in terms of quality are sufficiently high. The main consequence is that in the initial phase of market evolution, 'new' (i.e. without an installed base) customers continuously enter the market and 'second mover' manufacturers can cater for them. As a result competition level stays relatively higher than in the other two markets. As market 3 should be interpreted as the market for LAN switches, this result is in line with the observed evolution of the LAN industry as described in Section 2.3 above (see Table 3 in particular).

Concerning the effect of switching costs and compatibility on HHI, it can be noted that an increase in the level of switching costs (i.e. high value of $\sigma$ ) leads to a (small) increase in concentration in both market 1 and 2 irrespective of $\gamma$. This results follows from a switching cost related first mover advantage that affects the concentration level in two ways. On the one hand, there is a direct effect. Higher switching costs reduce competition and therefore increase concentration. On the other hand there is an indirect effect. Higher switching costs tend to favour M\&As also in the initial stages of industry evolution since, by assumption, mergers are more likely between firms with high shares (in different markets). As a result HHI (slightly) increases.

For given values of switching costs, changes in the degree of compatibility impact unevenly across markets. In particular, an increase in compatibility (i.e. higher value of $\gamma$ ) has ambiguous (and again small) effects on concentration levels in markets 1 and 2 as it increases concentration in market 1 when switching costs are low and decreases it otherwise. However, it increases concentration in market 3 . This is a result that can be explained in terms of the interplay between firms' strategies, demand heterogeneity, and complementarity among products. 
Consider first markets 1 and 2. Because of heterogeneous demand, customers will purchase those products that reflect their desired quality. When firms pursue a 'close' strategy they can increase the appeal of some of their products that otherwise would remain unsold by 'bundling' them together. As a result, demand for these products would increase together with manufacturers' profits. However, pursuing this strategy reduces the extent to what customers can mix and match products. This is likely to reduce demand for other products which can potentially cater for a larger share of customers. Going into opposite directions, the two effects tend to cancel out and, as a result, concentration level in the two markets is not clearly related to changes in $\gamma$.

Different is the case of market 3 in which pursuing a 'closer' strategy ends up reducing concentration. This result follows from the dynamic of entry into the new market embodied in our model. Indeed, one feature of our model is that start-ups are among the early entrants in the new market. Being first movers they are likely to exploit their advantage and grow. Incumbents can react to new entrants either by acquiring the new firms (see below) or by 'closing' their system. By reducing compatibility across firm, a 'close' system strategy allows incumbents to leverage upon their installed base in the other two markets and establish their share in the new one. As a result, the concentration level remains relatively low.

The market shares of the leader firm across periods and markets are reported in Table 7 below.

[Insert Table 7 about here]

These results mirror those just discussed. For a given scenario, the leader's shares increase over time in both market 1 and 2, while the leader in market 3 has a lower share. The impact of switching cost level and compatibility is the same as in the case of concentration.

To summarize, differences across the four scenarios exist, both in concentration levels and leader market shares. However, these differences are small, which suggest that results (and consequently their 'history-friendliness') are robust with respect to the propose variations.

\subsubsection{Results for industry dominance}

It is interesting to understand whether market concentration and leadership in one market is accompanied by leadership in a related market and to what extent this relationship depends on switching costs and compatibility. We report this information in Table 8 below. In particular, columns (1) and (2) report the frequency of runs in which a specific firm achieves the highest shares 
in every 'active' market (i.e. the markets in which at least one unit is sold) after 100 and 200 periods respectively. This is an indication of the 'dominance' of a specific firm in the LAN industry. This frequency can be interpreted as the probability that the real pattern (i.e. dominance) emerges out of the simulation. Columns (3) and (4) instead report the average (across firms) dispersion in firms' market shares. Dispersion was computed as the variance of shares across active markets.

[Insert Table 8 about here]

Table 8 shows that history replicating runs (in terms of the emergence of dominance) have a significant probability to be observed under all parameter configurations. These results provide further support for the validity of our appreciative theory.

As for the effect of parameters variations on the probability of industry dominance, the following considerations are in order. First and foremost, such probability tends to increase with $\gamma$. This result indicates that, for a given level of switching costs, firms pursuing an 'open strategy' are more likely to achieve industry dominance after the new market opens up. This result is more evident for low value of switching costs. At first sight this result is surprising. When firms pursue an open strategy, customers can easily mix and match products in their system and manufacturers are likely to confront with a much higher level of competition. As a consequence, we should expect a decline in the market shares and consequently a reduction in the probability that one firm achieves industry dominance. However, in this context firms can also benefit from a relatively higher level of demand which allows them to grow. In our setting, firms with relatively high but similar shares in different markets are more likely to merge. When $\gamma$ is low instead, firms operating in different markets are less likely to become similar in terms of shares. This follows from the fact that the relatively smaller size of the overall market makes firms less likely to grow. As a consequence M\&As among 'equals' across different markets are less likely to occur.

Second, the effect of switching costs level is generally limited, with the partial exception of low compatibility at period 200 where high switching costs favour the emergence of large firms that end up merging with a positive impact on concentration. The above explanations are consistent with the values of the average dispersion index, where the higher the index is the more dissimilar tend to be the firms' shares across markets. 


\title{
6.1.3. Further results
}

We now move to consider the effect of our scenarios on another set of aspects that are relevant for describing the evolution of the LAN industry. These results are reported in Table 9 below.

\author{
[Insert Table 9 about here]
}

Column (1) reports the frequency of runs in which the firm that was the first to introduce a product of type 3 was a start-up (i.e. no products in markets 1 and 2). Frequencies range between $72 \%$ and $8 \%$. In our model the fact that start-ups firms are those most likely to open a new market follows from the assumption that competing in the new market requires new and specific capabilities and that the probability that a firm introduces a product of a an existing type is increasing in the number of products of that type already offered by the firm. As a consequence, incumbents are more likely to exploit their capabilities in markets they are already active in rather than exploring new ones.

Column (2) reports the frequency of runs in which the start-up that pioneered a product of type 3 ends up being acquired and/or merged. Our results indicate that this event happens in almost every run independently of the scenario we are considering. Indeed, the very high probability that a type 3 product pioneer firm is later acquired is a consequence of the start-up being almost a monopolist in the market. Being among the few firms with specific capabilities to supply the new product, start-ups became likely targets for acquisitions especially for those incumbents with high shares in the other markets.

Column (3) reports the frequency of runs in which the start-up that starts the new market becomes the leader of the market at the time of the simulation horizon. This statistics includes the cases in which the start-up was acquired. Our results indicate that leadership is almost always achieved in every scenario. Incumbents enter the new market by acquisition and exploit their strength in at least one of the existing markets to extend their leadership also to the new market.

\subsection{History-divergent runs}

Our results in the previous section seem to well replicate the dynamics of innovation, entry and growth that have characterized the evolution of the LAN industry. When the switch market started as a result of technical change, start-ups firms were among the early entrants. These firms were usually mono product firms with high R\&D intensity and highly specific capabilities. Few of them, however, survived and were able to make it to the next round. Acquisition of new start-ups 
provided incumbents with the required knowledge to compete in the new market as well as with the necessary products to extend their portfolio. Once entered in the new market, incumbents used their dominant position in other markets to become dominant also in the new one.

As mentioned in Section 4, we follow the HFMs methodology and carry out counterfactual analyses. The first exercise we perform intends to disentangle the relative importance of the different mechanisms we identified as important to explain the industry evolution. We start by considering what happens in the absence of switching costs and full compatibility. These results are summarized in Table 10.

[Insert Table 10 about here]

Setting switching costs to zero has a very strong impact on industry evolution, leading to truly history-divergent results. Concentration levels in markets 1 and 2 are significantly lower than in our baseline case, even lower than concentration in market 3, which goes against the observed evidence. Moreover, dominance is significantly less likely to occur. The same result, however, does not happen when varying the degree of compatibility.

In their explanation of the evolution of the industry, Carpenter et al. (2003) pointed to the role of corporate stocks in the accumulation of the innovative capabilities necessary to compete in the optical networking industry during the second half of the 1990s. Though insightful, this explanation is however partial as it lacks an explanation of how Cisco Systems rose to dominance in the data networking systems for enterprises before challenging existing incumbents in the optical networking industry. In this respect, our review of the main events in the evolution of the industry during the 1990s, and the appreciative theory, has highlighted the important role played by the presence of switching costs and compatibility strategies. The counterfactual just presented further confirms the appreciative theory and highlight the important role played by switching costs in reproducing the high level of concentration and the dominance of a single firm. All in all, this result suggests that good calibration (i.e. 'history friendliness') seems to require the inclusion of demand side factors such as switching costs. ${ }^{14}$

\footnotetext{
${ }^{14}$ The importance of switching costs is also confirmed by an experiment (nor reported here) in which for each firm the switching cost parameter was positive with probability 0.5 , and null otherwise.. Out of 100 simulations, it turns out that $97 \%$ of the times a market leader emerges with positive switching costs in market $1,94 \%$ of the times in market 2 , and $67 \%$ of the times in market 3 .
} 
Concerning the role of compatibility, results are less clear-cut. Our model suggests that firms' strategies to 'close' the systems should combine with firm expertise in order to achieve high market shares and leadership. Indeed, in the absence of strong economies of scope among different products, as it is the case in our model, a strategy to 'close' the system alone does not provide any comparative advantage, as firms' lack of expertise and capabilities in one market makes the strategy ineffective. ${ }^{15}$

As a second counterfactual, we explore the extent to what M\&As, which we did observe in reality, played a crucial role for the consolidation of the industry into a tight oligopoly. In their case study of Cisco Systems' acquisition strategy, Mayer and Kenney (2004) argued that the development of an 'acquisition and development' strategy was essential for the survival and growth of the company and that acquisitions played an important role in its overall competitive strategy. To gain a better understanding on whether $M \& A s$ are crucial vis-à-vis other mechanisms we exclude in our counterfactual the possibility to merge. The results of this exercise are summarized in Table 11.

\section{[Insert Table 11 about here]}

The first result we observe concerns the concentration levels (see the upper panel). Apart from cases in which switching costs are high and compatibility is low, concentration is usually lower than in our baseline case. More interestingly, it does not grow when market 3 starts and even decreases in few cases. The second result is that no dominant firm in the industry seems to emerge at the end of the simulation runs. Both results point to the crucial role played by M\&As both in determining the competitive strategy at the firm level, as argued by Mayer and Kenney (2004), and in shaping the industry evolution. ${ }^{16}$

In the third counterfactual we further investigate the role played by demand heterogeneity. In our model we assume that customers have heterogeneous preferences and that, as a consequence, submarkets exist within each market corresponding to the specific needs of customer firms. This is what we observed in reality both in the case of routers (i.e. multiprotocol vs. access-routers) and in the case of switches (high-end vs. low-end). To understand the implication of this assumption for industry level dynamics our counterfactual assumes instead homogenous demand. In particular,

\footnotetext{
${ }^{16}$ It must be highlighted that M\&As may not be needed if switching costs are high and compatibility low. In this case, first mover advantages (in a market, then extended to others through 'closure') can be so strong to lead to dominance without mergers.
} 
in Equation (5.6), we fix $D_{i 0}=\frac{D}{2}$ for each $i$. The other parameters values remain set as in Scenario $_{(1.5-0.6-0.2)}$. The results of this exercise are reported in Table 12.

\section{[Insert Table 12 about here]}

Assuming homogeneous demand does not substantially change the main results of industry

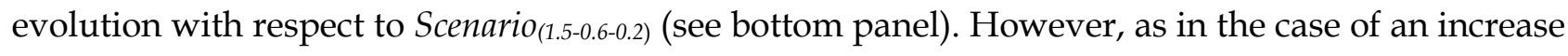
in switching costs, the level of concentration within each market slightly increases (see top panel).

As a final counterfactual, we explore an alternative mechanism to create first mover advantages based on the persistence in the innovative activity. We rule out any demand related mechanism, by setting switching costs to zero and imposing full compatibility, while at the same time we impose increasing returns at the market level by modifying the innovation rule as follows. In particular, we implement the following algorithm. First, with probability $p^{*} n$ (where $p$ is fixed at the level of the baseline simulation), there is one innovation. The innovation is given to firm $i$ with probability proportional to $1+n_{i}^{t-1}$ where $n_{i}^{t-1}$ is the total number of products (of all types) offered by firm $i$ at $t-1$. The probability is therefore:

$$
\operatorname{Pr}(" i \text { innovates" })=\frac{1+n_{i}^{t-1}}{\sum_{k=1}^{N}\left(1+n_{k}^{t-1}\right)}
$$

Once the product is assigned to a firm, the product type is determined as in (5.3). While there is little and unclear evidence that persistence in product innovation historically played an important role for achieving market leadership in the LAN industry ${ }^{17}$, we think this exercise could indirectly increase the support for our model by ruling out an alternative mechanism in principle able to account for the industry evolution.

Results, which are reported in Table 12 (bottom panel), show that this version of the model is able to reproduce all the observed features of industry evolution, with an exception. In fact, the concentration in market 3 (the switch market in reality) is higher than concentration in markets 1

\footnotetext{
17 Fontana et al. (2014) show that technological leaders in the LAN industry were more likely to be persistent product innovators in their market. However, they do not investigate the relationship between innovation persistence and market leadership.
} 
and 2, which is contrary to what can be observed in the history. The explanation of this counterfactual result is that the increasing return mechanism we impose favors incumbents (in the overall market) vis-à-vis new entrants, which have instead more opportunities to gain market share when the source of first-mover advantage is specific to a single market as in the case of switching costs.

\section{Discussion}

In Section 4 we argued that HFMs can (in a sense, should) provide insights that go beyond the specific case study investigated. This section we discuss how our results can be seen in this perspective.

First and foremost, our results contribute to the literature that investigates the conditions under which diversifying entrants may perform better than other types of entrants (de-novo entrants in particular) (Klepper and Simons, 2000; King and Tucci, 2002; Klepper, 2002) in an industry. Superior knowledge of both technology and market has been often identified as a fundamental driver (Agarwal et al., 2010) of this performance. Our results add to this literature by explicitly suggesting that mergers may also play an important role for the acquisition of superior technological and market know-how upon entry.

Second, our analysis contributes to the literature that looks at inter-markets relationships and, with specific reference to the HFM tradition, to the co-evolutionary dynamics of related markets (Malerba et al., 2008; Malerba and Orsenigo, 2010). Our results show that new entrants can exploit their installed base in a complementary market to obtain a superior performance in the new market whenever the possibility for customers to 'mix and match' products is somehow constrained. In addition to this, our model also shows that this performance gain in the new market may feedback into the firm's focal market and strengthen its position there. While the co-evolution of vertically related market has been already studied within the HFMs tradition (Malerba et al., 2008), to the best our knowledge the analysis of the co-evolution of complementary industries (or markets) had not yet been addressed. It can be noted that such co-evolutionary dynamics is at work in all those industries that can be characterized by the 'hardware-software' paradigm, such as the videogame and mobile phones industry.

Third, our results resonate with those of Bhaskarabhatla and Klepper (2014) who argue that in the laser industry, following technological change, the emergence of an 'integrative submarket' 
contributed to the convergence toward a particular design of the product which catered for the preference of different types of users (Windrum, 2005) and paved the way to the industry shakeout. The main difference is that while in the case of lasers, the integrative market was an 'old' existing niche, in the LAN case it was a new market (i.e. the one for switches). When this happens, entry by acquisition in the 'integrative submarket' (as done by Cisco Systems) might be the best strategy for incumbents to maintain and extend their leadership.

Finally, although the effect is not particularly strong, our results that the presence of demand heterogeneity tends to mitigate market concentration are consistent with some recent works on the dynamics of some industries characterized by the presence of sub-markets and absence of shakeouts (Klepper and Thompson, 2006; Buenstorf and Klepper, 2010).

From the methodological viewpoint, our findings may be relevant also for other modelling traditions, in particular within mainstream IO. First and foremost, they are relevant for the literature on switching costs (Klemperer, 1987; Matutes and Regibau, 1988 among others). Looking at firms strategic interactions and relying upon strong assumptions in terms of rationality, this literature predicts that in the presence of switching costs convergence in market shares is usually achieved, since incumbents have interest in keeping the price high in order to exploit their installed base, leaving 'unattached' consumers to new firms (Beggs and Klemperer, 1992). Our model considers instead the presence of switching costs in a context of uncertain innovation. It suggests that new firms may lack the technological capabilities to reduce the gap with incumbents which can instead use M\&As to consolidate their position when compatibility is partial. This conduct is likely to be beneficial for incumbents firms also in other industries where the outcome of innovation is uncertain and switching costs are paramount.

Second, our findings qualify some of the prior results in the literature on the market effects of compatibility. This literature traditionally posits the presence of a dichotomy between fully compatible (i.e. open) systems and fully incompatible (i.e. closed) systems. As we have argued in our appreciative theory, this dichotomy is hardly found in practice as firms may partially 'close' their systems even in the presence of common or open standards. Within this context, industry concentration may depend on the degree of compatibility rather than its mere presence (or lack thereof). It is likely that these findings are generalizable to other 'platform based' industries (i.e. semiconductors, mobile phones etc.) when the platform leaders have the choice about whether to open or close their system to third party contributors. 


\section{Conclusion}

This paper has proposed a history friendly model of the evolution of the LAN industry in the 1990s. Our model embodies some key features of the industry: competition in product innovation with demand heterogeneity, switching costs, the possibility of supplying 'close' systems, the emergence of new markets, and M\&As. Our results are able to reproduce some stylized facts of the industry. In the model, as in the 'real world', we observe a tendency towards the creation of a dominating firm (Cisco Systems in the reality). This firm starts from a dominant position in one of the existing markets (i.e. routers) and reinforces its position when a new market (i.e. switches) emerges. The new market is pioneered by a new start-up (Kalpana in the reality), which is soon acquired by the dominant incumbent (Cisco Systems). As a result of the acquisition, the acquiring firm becomes the leader also in the new market.

The limitations of our model can represent avenues for future research, also beyond a HF perspective. Here we briefly mention two of them, both going in the direction of endogenizing firms' strategies. First of all, innovation is modelled as an exogenous event. While this is a sensible choice on both theoretical and empirical grounds, a more complete treatment would probably require modelling the probability to innovate as a function of firm's $R \& D$ investment and, possibly, spillovers by other firms similarly to what has been done in Silverberg and Verspagen (1994). Also, in our model switching costs and compatibility were exogenously given. One possibility would be to introduce firm-specific levels of switching costs and compatibility by allowing simple (i.e. myopic) strategic reasoning in the model. 
Acknowledgements: we have greatly benefited from the comments of two anonymous referees, Shane Greenstein, the late Steven Klepper, William Lazonick, Franco Malerba. Preliminary versions of this paper have been presented at the 2006 Schumpeter Society Conference, at the October 2008 DIME workshop on 'Demand, Innovation, and Industrial Dynamics', and at a seminar at CIRCLE, Lund University, December 2012. The usual disclaimers apply. 


\section{REFERENCES}

Adner R, Levinthal D (2001) Demand heterogeneity and technology evolution: implications for product and process innovation. Management Science, 47 (5): 611-628.

Agarwal R, Echambadi R, Franco AM, and Sarkar MB (2004) Knowledge transfer through inheritance: Spin-out generation, development, and survival. Academy of Management Journal, 47(4), 501-522.

Beggs A., Klemperer P. (1992) Multi-period competition with switching costs. Econometrica, 60(3): 651-666.

Bhaskarabhatla A, Klepper S (2014) Latent submarket dynamics and industry evolution: lessons from the US laser industry. Industrial and Corporate Change, forthcoming.

Bottazzi G, Dosi, G., Rocchetti G. (2001) Modes of knowledge accumulation, entry regimes and patterns of industrial evolution. Industrial and Corporate Change, 10 (3), 609-638.

Buenstorf G, Klepper S (2010) Submarket dynamics and innovation: the case of the US tire industry. Industrial and Corporate Change, 19(5): 1563-1587.

Carpenter M, Lazonick W, O'Sullivan M (2003) The stock market and innovative capability of the New Economy: the optical networking industry. Industrial and Corporate Change, 12(5): 963-1034.

Chen P, Forman C (2006) Can vendors influence switching costs and compatibility in an environment with open standards? MIS Quarterly, 30: 1-22.

Christensen KJ, Haas LC, Noel FE, Strole NC (1995) Local area networks. Evolving from shared to switched access. IBM Systems Journal 34: 347-374

Cohen W, Klepper S (1996) Firm size and the nature of innovation within industries: the case of process and product R\&D. Review of Economics and Statistics, 78(2), 232-43. 
Cowan R., Foray, D. (2002) Evolutionary economics and the counterfactual threat: on the nature and role of counterfactual history as an empirical tool in economics. Journal of Evolutionary Economics 12(5): 539-562.

Cowan R, Jonard N, Zimmerman JB (2006) Evolving networks of inventors. Journal of Evolutionary Economics, 16: 155-174.

Doms M (2003) Communications equipment: what has happened to prices? Federal Reserve Bank of San Francisco, Working Paper $n^{\circ} 15$, June.

Elster, J (1978). Logic and society: Contradictions and possible worlds. New York: Wiley.

Fagiolo G, Moneta A, Windrum, P. (2007) A critical guide to empirical validation of agent-based models in economics: Methodologies, procedures, and open problems. Computational Economics 30 (3): 195-226.

Farrell J, Shapiro C (1988) Dynamic competition with switching costs. RAND Journal of Economics 19: 123-137.

Fontana R (2008) Competing technologies and market dominance: standard 'battles' in the Local Area Networking industry, Industrial and Corporate Change, 17(6): 1205-1238.

Fontana R, Moriniello D and Vezzulli, A. (2014) An empirical study of technological leadership and persistence in product innovation, mimeo.

Fontana R, Nesta L (2006) Product entry in a fast growing industry: the LAN switch market, Journal of Evolutionary Economics, 16(1-2), 45-64.

Fontana R, Nesta L (2009) Product innovation and survival in a high-tech industry. Review of Industrial Organization, 34(4), 287-306. 
Garavaglia C. (2010). Modelling industrial dynamics with "History-friendly" simulations. Structural Change and Economic Dynamics, 21(4); 258-275.

Garavaglia C., Malerba F., Orsenigo L., Pezzoni M. (2012). Technological regimes and demand structure in the evolution of the pharmaceutical industry. Journal of Evolutionary Economics, 22 (4) 4, 677-709.

Gawer A, Cusumano M (2002) Platform leadership: how Intel, Microsoft and Cisco drive industry innovation. Harvard University Press, Boston

Jain S (2012) Pragmatic agency in technology standards setting: The case of Ethernet. Research Policy, 41(9), 1643-1654.

Kim C, Lee, K (2003) Innovation, technological regimes and organizational selection in industry evolution: a 'history friendly model' of the DRAM industry. Industrial and Corporate Change, 12(6): 1195-1221.

King AA, Tucci CL (2002) Incumbent entry into new market niches: The role of experience and managerial choice in the creation of dynamic capabilities. Management Science, 48(2): 171-186.

Klemperer P (1987) Markets with consumer switching costs. Quarterly Journal of Economics, 102(2): 375-394.

Klepper S (2002) The capabilities of new firms and the evolution of the US automobile industry. Industrial and corporate change, 11(4), 645-666.

Klepper S and Simons KL (2000) Dominance by birthright: entry of prior radio producers and competitive ramifications in the US television receiver industry. Strategic Management Journal, 21(10-11), 997-1016.

Klepper S and Thompson P (2006) Submarkets and the evolution of market structure. RAND Journal of Economics, 37: 861-886.

Lewis D. (1973), Counterfactuals. Oxford: B. Blackwell. 
Malerba, F. (2002). Sectoral systems of innovation and production. Research policy, 31(2): 247-264.

Malerba F, Nelson R, Orsenigo L, Winter S (1999) History friendly models of industry evolution: the computer industry. Industrial and Corporate Change, 1, 3-41.

Malerba F, Nelson R, Orsenigo L, Winter S (2008) Vertical integration and disintegration of computer firms: a history-friendly model of the coevolution of the computer and semiconductor industries. Industrial and Corporate Change, 17(2):197-231.

Malerba F, Nelson R, Orsenigo L, Winter S (2007) Demand, innovation, and the dynamics of market structure: The role of experimental users and diverse preferences. Journal of Evolutionary Economics, 17(4): 371-399.

Malerba, F., Nelson, R., Orsenigo, L., Winter, S. (2001). Competition and industrial policies in a 'history friendly' model of the evolution of the computer industry. International Journal of Industrial Organization, 19(5):635-664.

Malerba, F. , Orsenigo, L. (1996). The dynamics and evolution of industries. Industrial and Corporate Change, 5(1): 51-87.

Malerba F, Orsenigo L. (2002) Innovation and market structure in the dynamics of the pharmaceutical industry and biotechnology: towards a history-friendly model. Industrial and Corporate Change, 11(4), 667-704.

Malerba, F., Orsenigo, L. (2010). User-producer relations, innovation and the evolution of market structures under alternative contractual regimes. Structural Change and Economic Dynamics, 21(1): 26-40.

Miller M (1994) Routers: poised to make the transition. Network World, September , 77-94

Miller M (1996) Routers take new root. Network World, July, 43-50

Matutes C, Regibeau P (1988) 'Mix and match': product compatibility without network externalities. RAND Journal of Economics, 19(2): 221-234. 
Mayer D, Kenney M (2004) Economic action does not take place in a vacuum: understanding Cisco's acquisition and development strategy. Industry and Innovation, 11(4): 299-325.

Paulsen, E. (2001), Inside Cisco. The real story of sustained MEA growth. New York, Wiley and Sons

Silverberg G, B Verspagen (1994) Learning, innovation and economic growth. A long run model of industrial dynamics, Industrial and Corporate Change, 3, 199-224.

Tucker, A. (1999). Historiographical counterfactuals and historical contingency. History and Theory, 38(2), 264-276.

von Burg U (2001) The triumph of Ethernet. Stanford, Stanford University Press.

Weisbuch G, Kirman A, Herreiner D. (2000) Market organisation and trading relationships. The Economic Journal, 110 (463): 411-436.

Windrum, P. (2005), 'Heterogeneous preferences and new innovation cycles in mature industries: the amateur camera industry 1955-1974,' Industrial and Corporate Change, 14: 1043-1074.

Windrum P, Birchenhall C (1998) Is product life cycle theory a special case? Dominant designs and the emergence of market niches through coevolutionary learning. Structural Change and Economic Dynamics, 9 9(1): 109-134.

Winter, S. G., Kaniovski, Y. M., \& Dosi, G. (2003). A baseline model of industry evolution. Journal of Evolutionary Economics, 13(4): 355-383. 
FIGURE1. LAN INDUSTRY. REVENUES BY MARKET

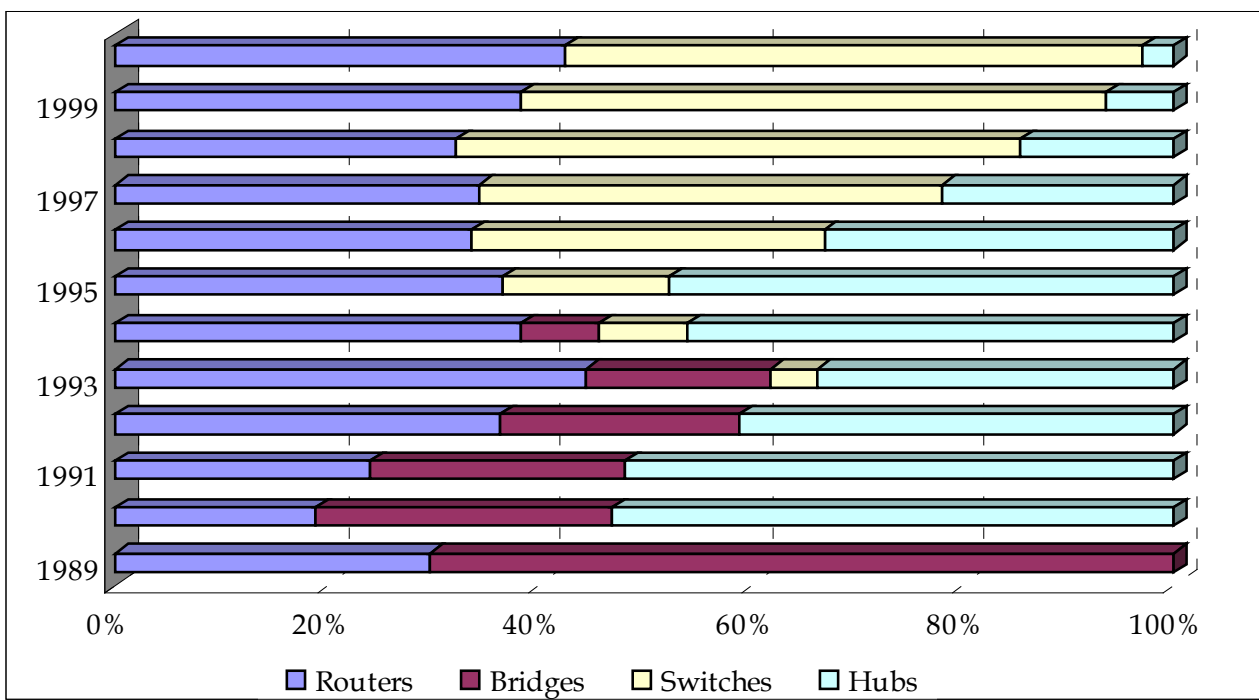


LIST OF TABLES

TABLE 1. ROUTER MARKET: REVENUES' MARKET SHARES

\begin{tabular}{|c|c|c|c|c|c|c|c|}
\hline 1990 & & 1993 & & 1995 & & 1997 & \\
\hline$\underline{\text { Vendor }}$ & $\underline{\%}$ & $\underline{\text { Vendor }}$ & $\underline{\%}$ & $\underline{\text { Vendor }}$ & $\underline{\%}$ & $\underline{\text { Vendor }}$ & $\underline{\%}$ \\
\hline Cisco Systems & 35.2 & Cisco Systems & 50 & Cisco Systems & 48 & Cisco Systems & 63 \\
\hline DEC & 20.3 & WellFleet Comm. & 16 & Bay Networks & 17 & Bay Networks & 10 \\
\hline 3Com & 13.2 & IBM & 7 & Motorola & 6 & 3Com & 4 \\
\hline WellFleet Comm. & 8.7 & 3Com & 6 & 3Com & 5 & Motorola & 3.5 \\
\hline Proteon & 7.8 & DEC & 4 & IBM & 3 & IBM & 2.2 \\
\hline Others & 14.8 & Others & 17 & Others & 21 & Others & 17.3 \\
\hline TOTAL & 100 & TOTAL & 100 & TOTAL & 100 & TOTAL & 100 \\
\hline
\end{tabular}

SOURCE; AUTHORS' ELABORATION BASED ON DELL'ORO GROUP REPORTS 
TABLE 2. ROUTER MARKET: REVENUES' MARKET SHARES by SUB-MARKET

\begin{tabular}{|c|c|c|c|c|c|c|c|}
\hline \multicolumn{4}{|c|}{ MULTI-PROTOCOL SUB-MARKET } & \multicolumn{4}{|c|}{ REMOTE ACCESS SUB-MARKET } \\
\hline$\underline{1996}$ & & $\underline{1997}$ & & $\underline{1996}$ & & $\underline{1997}$ & \\
\hline$\underline{\text { Vendor }}$ & $\underline{\%}$ & $\underline{\text { Vendor }}$ & $\underline{\%}$ & $\underline{\text { Vendor }}$ & $\underline{\%}$ & $\underline{\text { Vendor }}$ & $\underline{\%}$ \\
\hline Cisco Systems & 64 & Cisco Systems & 58 & US Robotics & 26.1 & 3Com Corp.* & 34 \\
\hline Bay Networks & 10 & Bay Networks & 12.7 & Ascend & 22.7 & Ascend & 27 \\
\hline 3Com Corp. & 4 & Motorola & 5.5 & Cisco Systems & 11.4 & Cisco Systems & 18 \\
\hline IBM & 2 & 3Com Corp. & 4.6 & Shiva & 8.2 & Shiva & 5 \\
\hline Motorola & 2 & IBM & 2 & 3Com Corp. & 6.2 & US Robotics & - \\
\hline Others & 18 & Others & 17.2 & Others & 25.4 & Others & 16 \\
\hline TOTAL & 100 & TOTAL & 100 & TOTAL & 100 & TOTAL & 100 \\
\hline
\end{tabular}

* 3COM CORP. ACQUIRED US ROBOTICS IN 1997

SOURCE; AUTHORS' ELABORATION BASED ON DELL'ORO GROUP REPORTS 
TABLE 3. SWITCH MARKET: REVENUES' MARKET SHARES

\begin{tabular}{lcllc}
\hline \multicolumn{1}{c}{1994} & & \multicolumn{2}{c}{$1999(2 \mathrm{qt})$} \\
\hline$\underline{\text { Vendor }}$ & $\underline{\%}$ & $\underline{\text { Vendor }}$ & $\underline{\%}$ \\
Kalpana & 53 & Cisco Systems Inc. & 46.7 \\
3Com Corp. (Synernetics) & 29 & 3Com Corp. & 13.1 \\
Alantec Corp. & 7 & Nortel Networks & 11.1 \\
Chipcom Corp. & 5 & Cabletron Systems Inc. & 9.8 \\
Lannet & 3 & IBM & 4 \\
\hline \multicolumn{1}{c}{ TOTAL } & 100 & & TOTAL & 100
\end{tabular}

SOURCES: 1994: THE YANKEE GROUP; 1999: DELL'ORO GROUP REPORTS 
TABLE 4. TOP 5 COMPANIES IN TERMS OF DISTANCE FROM TECHNOLOGICAL FRONTIER

\begin{tabular}{|c|c|c|c|c|}
\hline \multicolumn{5}{|c|}{ ROUTERS } \\
\hline RANK & 1993 & 1995 & 1997 & 1999 \\
\hline 1 & Wellfleet Comm. & Newbridge Networks & Newbridge Networks & Juniper Networks \\
\hline 2 & RAD Network Devices & 3Com Corp. & Xyplex Whittaker & Cisco Systems \\
\hline 3 & Cisco Systems & Network Systems Corp. & Cisco Systems & 3Com Corp. \\
\hline 4 & ACC & Cray Communications & 3Com Corp. & NeoNet-works \\
\hline 5 & Andrew Corp. & CrossComm Corp. & Network Systems Corp. & Cabletron Systems \\
\hline \multicolumn{5}{|c|}{ SWITCHES } \\
\hline RANK & 1993 & 1995 & 1997 & 1999 \\
\hline 1 & Fore Systems Inc. & Newbridge Networks & Newbridge Networks & Cisco Systems \\
\hline 2 & DEC & 3Com Corp. & Plaintree Systems & 3Com Corp. \\
\hline 3 & UB Networks Inc. & Hughes LAN Systems & Hughes LAN Systems & Fore Systems Inc. \\
\hline 4 & Grand Junction Networks & Cabletron Systems & Optical Data Systems & Lucent Networks. \\
\hline 5 & Hewlett-Packard Co. & IBM & Cabletron Systems & Foundry Networks \\
\hline
\end{tabular}

SOURCES: PREDICTED PRICES FROM HEDONIC REGRESSIONS BASED ON AUTHOR'S PRODUCT DATABASE 
TABLE 5. Possible SCENARIOS

\begin{tabular}{|c|c|c|c|c|}
\hline & & SWITCHING COSTS & COMPATIBILITY & M\&AS \\
\hline & \multicolumn{4}{|c|}{ History-replicating runs } \\
\hline 1 & SCENARIO $_{(1.5-0.2-0.2)}$ & Low $(\sigma=1.5)$ & $\operatorname{Low}(\gamma=0.2)$ & Allowed $(\theta=0.2)$ \\
\hline 2 & SCENARIO $_{(3-0.2-0.2)}$ & High $(\sigma=3)$ & Low $(\gamma=0.2)$ & Allowed $(\theta=0.2)$ \\
\hline 3 & SCENARIO $_{(1.5-0.6-0.2)}$ & Low $(\sigma=1.5)$ & $\operatorname{High}(\gamma=0.6)$ & Allowed $(\theta=0.2)$ \\
\hline \multirow[t]{2}{*}{4} & SCENARIO $_{(3-0.6-0.2)}$ & $\operatorname{High}(\sigma=3)$ & $\operatorname{High}(\gamma=0.6)$ & Allowed $(\theta=0.2)$ \\
\hline & \multicolumn{4}{|c|}{ History-divergent runs } \\
\hline 5 & SCENARIO $_{(1.5-1-0.2)}$ & Low $(\sigma=1.5)$ & Full $(\gamma=1)$ & Allowed $(\theta=0.2)$ \\
\hline 6 & SCENARIO $_{(3-1-0.2)}$ & $\operatorname{High}(\sigma=3)$ & Full $(\gamma=1)$ & Allowed $(\theta=0.2)$ \\
\hline 7 & SCENARIO $_{(0-0.2-0.2)}$ & Null $(\sigma=0)$ & $\operatorname{Low}(\gamma=0.2)$ & Allowed $(\theta=0.2)$ \\
\hline 8 & SCENARIO $_{(0-0.6-0.2)}$ & Null $(\sigma=0)$ & $\operatorname{High}(\gamma=0.6)$ & Allowed $(\theta=0.2)$ \\
\hline 9 & $\operatorname{SCENARIO}_{(0-1-0.2)}$ & Null $(\sigma=0)$ & Full $(\gamma=1)$ & Allowed $(\theta=0.2)$ \\
\hline 10 & SCENARIO $_{(1.5-0.2-0)}$ & Low $(\sigma=1.5)$ & $\operatorname{Low}(\gamma=0.2)$ & Not Allowed $(\theta=0)$ \\
\hline 11 & SCENARIO $_{(3-0.2-0)}$ & $\operatorname{High}(\sigma=3)$ & $\operatorname{Low}(\gamma=0.2)$ & Not Allowed $(\theta=0)$ \\
\hline 12 & SCENARIO $_{(1.5-0.6-0)}$ & Low $(\sigma=1.5)$ & High $(\gamma=0.6)$ & Not Allowed $(\theta=0)$ \\
\hline 13 & $\operatorname{SCENARIO}_{(3-0.6-0)}$ & $\operatorname{High}(\sigma=3)$ & $\operatorname{High}(\gamma=0.6)$ & Not Allowed $(\theta=0)$ \\
\hline 14 & SCENARIO $_{(\mathrm{H})}$ & Low $(\sigma=1.5)$ & $\operatorname{High}(\gamma=0.6)$ & Allowed $(\theta=0.2)$ \\
\hline 15 & SCENARIO $_{(\mathrm{I})}$ & Null $(\sigma=1.5)$ & Full $(\gamma=1)$ & Allowed $(\theta=0.2)$ \\
\hline
\end{tabular}


TABLE 6. MARKET CONCENTRATION (HERFINDAHL HIRSCHMANN INDEXES)

\begin{tabular}{lccccc}
\hline & $\mathrm{HHI}_{(1-100)}$ & $\mathrm{HHI}_{(1-200)}$ & $\mathrm{HHI}_{(2-100)}$ & $\mathrm{HHI}_{(2-200)}$ & $\mathrm{HHI}_{(3-200)}$ \\
\hline SCENARIO $_{(1.5-0.6-0.2)}$ & 0.478 & 0.605 & 0.433 & 0.545 & 0.339 \\
SCENARIO $_{(1.5-0.2-0.2)}$ & 0.430 & 0.545 & 0.444 & 0.554 & 0.245 \\
SCENARIO $_{(3-0.6-0.2)}$ & 0.498 & 0.636 & 0.441 & 0.589 & 0.338 \\
SCENARIO $_{(3-0.2-0.2)}$ & 0.508 & 0.672 & 0.480 & 0.623 & 0.258 \\
\hline
\end{tabular}


TABLE 7. LEADER MANUFACTURER (MARKET SHARES)

\begin{tabular}{|c|c|c|c|c|c|}
\hline & $\operatorname{LEADER}_{(1-100)}$ & $\operatorname{LEADER}_{(1-200)}$ & $\operatorname{LEADER}_{(2-100)}$ & $\operatorname{LEADER}_{(2-200)}$ & $\operatorname{LEADER}_{(3-200)}$ \\
\hline SCENARIO $_{(1.5-0.6-0.2)}$ & 0.619 & 0.727 & 0.565 & 0.670 & 0.464 \\
\hline $\operatorname{SCENARIO}_{(1.5-0.2-0.2)}$ & 0.575 & 0.673 & 0.585 & 0.682 & 0.366 \\
\hline $\operatorname{SCENARIO}_{(3-0.6-0.2)}$ & 0.631 & 0.745 & 0.567 & 0.705 & 0.515 \\
\hline $\operatorname{SCENARIO}_{(3-0.2-0.2)}$ & 0.641 & 0.787 & 0.613 & 0.738 & 0.379 \\
\hline
\end{tabular}


TABLE 8. INDUSTRY DOMINANCE (FREQUENCY) AND AVERAGE MARKET SHARE DISPERSION

\begin{tabular}{lcccc}
\hline & DOM. T $=100$ & DOM. T $=200$ & AV. DISP. T $=100$ & AV. DISP. T $=200$ \\
\hline SCENARIO(1.5-0.6-0.2) & 0.88 & 0.76 & 0.26 & 0.26 \\
SCENARIO(1.5-0.2-0.2) $_{\text {SCENARIO(3-0.6-0.2) }}$ & 0.8 & 0.54 & 0.27 & 0.33 \\
SCENARIO(3-0.2-0.2) $^{-0.76}$ & 0.76 & 0.76 & 0.37 & 0.23 \\
\hline
\end{tabular}


TABLE 9. ADDiTIONAL STATISTICS

\begin{tabular}{lccc}
\hline & INN3NEW & INN3MERGE & INN3LEADER \\
\hline SCENARIO(1.5-0.6-0.2) & 0.76 & 0.96 & 0.96 \\
$\operatorname{SCENARIO}_{(1.5-0.2-0.2)}$ & 0.88 & 0.92 & 0.96 \\
$\operatorname{SCENARIO}_{(3-0.6-0.2)}$ & 0.72 & 0.94 & 1 \\
SCENARIO$_{(3-0.2-0.2)}$ & 0.76 & 0.98 & 1 \\
\hline
\end{tabular}


TABLE 10 COUNTERFACTUAL \#1: NO SWITCHING COSTS AND FULL COMPATIBILITY

\begin{tabular}{|c|c|c|c|c|c|}
\hline \multirow{4}{*}{$\operatorname{SCENARIO}_{(1.5-1-0.2)}$} & $\mathrm{HHI}_{(1-100)}$ & $\mathrm{HHI}_{(1-200)}$ & $\mathrm{HHI}_{(2-100)}$ & $\mathrm{HHI}_{(2-100)}$ & $\mathrm{HHI}_{(3-100)}$ \\
\hline & 0.435 & 0.593 & 0.410 & 0.561 & 0.374 \\
\hline & INN3NEW & INN3LEADER & $\operatorname{LEADER}_{(3-200)}$ & INN3MERGE & DOM. $\mathrm{T}=200$ \\
\hline & 0.6 & 1 & 0.504 & 0.94 & 0.72 \\
\hline \multirow{4}{*}{ SCENARIO $_{(3-1--0.2)}$} & $\mathrm{HHI}_{(1-100)}$ & $\mathrm{HHI}_{(1-200)}$ & $\mathrm{HHI}_{(2-100)}$ & $\mathrm{HHI}_{(2-100)}$ & $\mathrm{HHI}_{(3-100)}$ \\
\hline & 0.491 & 0.671 & 0.443 & 0.622 & 0.354 \\
\hline & INN3NEW & INN3LEADER & $\operatorname{LEADER}_{(3-200)}$ & INN3MERGE & DOM. $\mathrm{T}=200$ \\
\hline & 0.84 & 0.96 & 0.493 & 0.96 & 0.88 \\
\hline \multirow{4}{*}{$\operatorname{SCENARIO}_{(0-0.2-0.2)}$} & $\mathrm{HHI}_{(1-100)}$ & $\mathrm{HHI}_{(1-200)}$ & $\mathrm{HHI}_{(2-100)}$ & $\mathrm{HHI}_{(2-100)}$ & $\mathrm{HHI}_{(3-100)}$ \\
\hline & 0.134 & 0.204 & 0.130 & 0.189 & 0.294 \\
\hline & INN3NEW & INN3LEADER & $\operatorname{LEADER}_{(3-200)}$ & INN3MERGE & DOM. $\mathrm{T}=200$ \\
\hline & 0.96 & 1 & 0.399 & 0.98 & 0.32 \\
\hline \multirow{4}{*}{$\operatorname{SCENARIO}_{(0-0.6-0.2)}$} & $\mathrm{HHI}_{(1-100)}$ & $\mathrm{HHI}_{(1-200)}$ & $\mathrm{HHI}_{(2-100)}$ & $\mathrm{HHI}_{(2-100)}$ & $\mathrm{HHI}_{(3-100)}$ \\
\hline & 0.142 & 0.202 & 0.139 & 0.209 & 0.360 \\
\hline & INN3NEW & INN3LEADER & $\operatorname{LEADER}_{(3-200)}$ & INN3MERGE & DOM. $\mathrm{T}=200$ \\
\hline & 0.84 & 1 & 0.468 & 1 & 0.38 \\
\hline \multirow{4}{*}{$\operatorname{SCENARIO}_{(0-1-0.2)}$} & $\mathrm{HHI}_{(1-100)}$ & $\mathrm{HHI}_{(1-200)}$ & $\mathrm{HHI}_{(2-100)}$ & $\mathrm{HHI}_{(2-100)}$ & $\mathrm{HHI}_{(3-100)}$ \\
\hline & 0.137 & 0.197 & 0.136 & 0.191 & 0.363 \\
\hline & INN3NEW & INN3LEADER & $\operatorname{LEADER}_{(3-200)}$ & INN3MERGE & DOM. $\mathrm{T}=200$ \\
\hline & 0.8 & 1 & 0.464 & 0.94 & 0.42 \\
\hline
\end{tabular}


TABLE 11. COUNTERFACTUAL \#2: NO MERGERS

\begin{tabular}{|c|c|c|c|c|c|}
\hline \multirow{5}{*}{$\operatorname{SCENARIO}_{(1.5-0.2-0)}$} & $\mathrm{HHI}_{(1-100)}$ & $\mathrm{HHI}_{(1-200)}$ & $\mathrm{HHI}_{(2-100)}$ & $\mathrm{HHI}_{(2-100)}$ & $\mathrm{HHI}_{(3-100)}$ \\
\hline & 0.359 & 0.368 & 0.413 & 0.410 & 0.328 \\
\hline & & & & & \\
\hline & INN3NEW & INN3LEADER & $\operatorname{LEADER}_{(3-200)}$ & DOM.T. $=100$ & DOM. $\mathrm{T}=200$ \\
\hline & 0.72 & 1 & 0.467 & 0 & 0 \\
\hline \multirow{4}{*}{ SCENARIO $_{(3-0.2-0)}$} & $\mathrm{HHI}_{(1-100)}$ & $\mathrm{HHI}_{(1-200)}$ & $\mathrm{HHI}_{(2-100)}$ & $\mathrm{HHI}_{(2-100)}$ & $\mathrm{HHI}_{(3-100)}$ \\
\hline & 0.508 & 0.672 & 0.480 & 0.623 & 0.258 \\
\hline & INN3NEW & INN3LEADER & $\operatorname{LEADER}_{(3-200)}$ & DOM.T. $=100$ & DOM. $\mathrm{T}=200$ \\
\hline & 0.82 & 1 & 0.441 & 0.02 & 0 \\
\hline \multirow{4}{*}{$\operatorname{SCENARIO}_{(1.5-0.6-0)}$} & $\mathrm{HHI}_{(1-100)}$ & $\mathrm{HHI}_{(1-200)}$ & $\mathrm{HHI}_{(2-100)}$ & $\mathrm{HHI}_{(2-100)}$ & $\mathrm{HHI}_{(3-100)}$ \\
\hline & 0.360 & 0.362 & 0.442 & 0.370 & 0.286 \\
\hline & INN3NEW & INN3LEADER & $\operatorname{LEADER}_{(3-200)}$ & DOM.T. $=100$ & DOM. $\mathrm{T}=200$ \\
\hline & 0.80 & 0.92 & 0.418 & 0 & 0 \\
\hline \multirow{5}{*}{$\operatorname{SCENARIO}_{(3-0.6-0)}$} & $\mathrm{HHI}_{(1-100)}$ & $\mathrm{HHI}_{(1-200)}$ & $\mathrm{HHI}_{(2-100)}$ & $\mathrm{HHI}_{(2-100)}$ & $\mathrm{HHI}_{(3-100)}$ \\
\hline & 0.376 & 0.386 & 0.415 & 0.416 & 0.274 \\
\hline & & & & & \\
\hline & INN3NEW & INN3LEADER & $\operatorname{LEADER}_{(3-200)}$ & DOM.T. $=100$ & DOM. $\mathrm{T}=200$ \\
\hline & 0.9 & 0.98 & 0.394 & 0.04 & 0 \\
\hline
\end{tabular}


TABLE 12. COUNTERFACTUAL \#3: HOMOGENEOUS DEMAND AND SUPPLY-RELATED INCREASING RETURNS

\begin{tabular}{|c|c|c|c|c|c|}
\hline \multirow{4}{*}{ SCENARIO $_{(\mathrm{H})}$} & $\mathrm{HHI}_{(1-100)}$ & $\mathrm{HHI}_{(1-200)}$ & $\mathrm{HHI}_{(2-100)}$ & $\mathrm{HHI}_{(2-100)}$ & $\mathrm{HHI}_{(3-100)}$ \\
\hline & 0.481 & 0.654 & 0.442 & 0.628 & 0.344 \\
\hline & INN3NEW & INN3LEADER & $\operatorname{LEADER}_{(3-200)}$ & INN3MERGE & DOM. $\mathrm{T}=200$ \\
\hline & 0.80 & 0.92 & 0.470 & 0.98 & 0.76 \\
\hline \multirow{5}{*}{ SCENARIO $_{(\mathrm{I})}$} & $\mathrm{HHI}_{(1-100)}$ & $\mathrm{HHI}_{(1-200)}$ & $\mathrm{HHI}_{(2-100)}$ & $\mathrm{HHI}_{(2-100)}$ & $\mathrm{HHI}_{(3-100)}$ \\
\hline & 0.266 & 0.390 & 0.260 & 0.395 & 0.477 \\
\hline & & & & & ח \\
\hline & INN3NEW & INN3LEADER & $\operatorname{LEADER}_{(3-200)}$ & INN3MERGE & DOM. $\mathrm{T}=200$ \\
\hline & 0.86 & 1 & 0.652 & 0.98 & 0.66 \\
\hline
\end{tabular}


APPENDIX

TABLE A1. NUMERICAL VALUES FOR INVARIANT PARAMETERS

\begin{tabular}{lll}
\hline PARAMETER & SYMBOL & VALUE \\
\hline Max responsivess to qualty & $\bar{D}$ & 0.6 \\
Growth in responsiveness to quality & $g$ & 0.01 \\
Min responsivess to qualty & $\underline{D}$ & 0.3 \\
Probability of changing LAN & $\mu$ & 0.1 \\
Number of customers & $\mathrm{N}^{\circ}$ customers & 1000 \\
Discrimination rate in consumer choice & $\lambda_{2}$ & 20 \\
Weight of product 1 in architecture A & $\alpha$ & 0.3 \\
Weight of product 1 in architecture B & $\beta_{1}$ & 0.1 \\
Weight of product 2 in architecture B & $\beta_{2}$ & 0.2 \\
Technological frontier for product 1 & frontier & \\
Technological frontier for product 2 & frontier $_{2}$ & 0.4 \\
Technological frontier for product 3 & frontier $_{3}$ & 0.4 \\
Probability of innovation & $p$ & 0.015 \\
Discrimination rate in product type choice & $\lambda_{1}$ & 0.3 \\
Propensity to merge & $\theta$ & 0.2 \\
Min probability of merger & $\Delta s_{\text {min }}$ & 0.1 \\
Number of manufacturers & $\mathrm{N}^{\circ}$ manufacturers & 30 \\
\hline
\end{tabular}




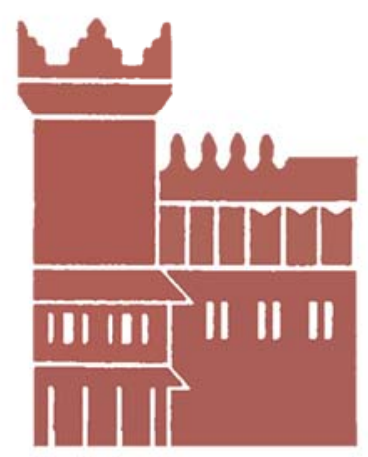

Alma Mater Studiorum - Università di Bologna DEPARTMENT OF ECONOMICS

Strada Maggiore 45

40125 Bologna - Italy

Tel. +39051 2092604

Fax +390512092664

http://www.dse.unibo.it 